\title{
BMJ Open Home and Online Management and Evaluation of Blood Pressure (HOME BP) digital intervention for self- management of uncontrolled, essential hypertension: a protocol for the randomised controlled HOME BP trial
}

\author{
Rebecca Band, ${ }^{1}$ Katherine Morton, ${ }^{1}$ Beth Stuart, ${ }^{2}$ James Raftery, ${ }^{3}$ \\ Katherine Bradbury, ${ }^{1}$ Guiqing Lily Yao, ${ }^{3}$ Shihua Zhu, ${ }^{3}$ Paul Little, ${ }^{2}$ Lucy Yardley, ${ }^{1}$ \\ Richard J McManus ${ }^{4}$
}

To cite: Band R, Morton $\mathrm{K}$, Stuart B, et al. Home and Online Management and Evaluation of Blood Pressure (HOME BP) digital intervention for selfmanagement of uncontrolled, essential hypertension: a protocol for the randomised controlled HOME BP trial. BMJ Open 2016;6: 012684. doi:10.1136/bmjopen-2016012684

- Prepublication history for this paper is available online To view these files please visit the journal online (http://dx.doi.org/10.1136/ bmjopen-2016-012684).

Received 17 May 2016

Revised 7 October 2016

Accepted 10 October 2016

CrossMark

For numbered affiliations see end of article.

Correspondence to

Dr Rebecca Band;

r.j.band@soton.ac.uk

\section{ABSTRACT}

Introduction: Self-management of hypertension, including self-monitoring and antihypertensive medication titration, lowers blood pressure (BP) at 1 year compared to usual care. The aim of the current trial is to assess the effectiveness of the Home and Online Management and Evaluation of Blood Pressure (HOME BP) intervention for the self-management of hypertension in primary care.

Methods and analysis: The HOME BP trial will be a randomised controlled trial comparing BP selfmanagement-consisting of the HOME BP online digital intervention with self-monitoring, lifestyle advice and antihypertensive drug titration - with usual care for people with uncontrolled essential hypertension. Eligible patients will be recruited from primary care and randomised to usual care or to self-management using HOME BP. The primary outcome will be the difference in mean systolic $\mathrm{BP}(\mathrm{mm} \mathrm{Hg})$ at 12-month follow-up between the intervention and control groups adjusting for baseline BP and covariates. Secondary outcomes (also adjusted for baseline and covariates where appropriate) will be differences in mean BP at 6 months and diastolic BP at 12 months; patient enablement; quality of life, and economic analyses including all key resources associated with the intervention and related services, adopting a broad societal perspective to include NHS, social care and patient costs, considered within trial and modelled with a lifetime horizon. Medication beliefs, adherence and changes; self-efficacy; perceived side effects and lifestyle changes will be measured for process analyses. Qualitative analyses will explore patient and healthcare professional experiences of HOME BP to gain insights into the factors affecting acceptability, feasibility and adherence.

Ethics and dissemination: This study has received NHS ethical approval (REC reference 15/SC/0082). The findings from HOME BP will be disseminated widely

\section{Strengths and limitations of this study}

- The study develops existing efficacious antihypertensive titration procedures into an online format.

- Home and Online Management and Evaluation of Blood Pressure (HOME BP) combines medication intensification with lifestyle change and behavioural support.

- The study has been designed to facilitate implementation within a primary care context.

through peer-reviewed publications, scientific conferences and workshops. If successful, HOME BP will be directly applicable to UK primary care management of hypertension.

Trial registration number: ISRCTN13790648; pre-results.

\section{INTRODUCTION}

Blood pressure (BP) is a key risk factor for cardiovascular disease, the largest cause of morbidity and mortality worldwide. ${ }^{1}$ The health survey for England (2012) identified that $\sim 31 \%$ of men and $27 \%$ of women in England have hypertension, ${ }^{2}$ yet over onethird of all treated patients currently fail to reach national guidelines for BP control. ${ }^{3}$ The mean $\mathrm{BP}$ in the UK currently ranks among the highest in Europe (27th/35), and the benefits of a population-wide reduction to levels seen in countries such as Denmark and Spain (which are among the lowest) would mean a $10 \mathrm{~mm} \mathrm{Hg}$ systolic blood pressure (SBP) reduction. ${ }^{4}$ A $10 \mathrm{~mm} \mathrm{Hg}$ 
reduction in BP is estimated to lead to a $41 \%$ reduction in stroke and a $22 \%$ reduction in coronary heart disease (CHD). ${ }^{5}$ New strokes affect over 120000 patients per annum in the UK, and stroke is the third leading cause of death and the most important cause of adult disability, representing $4.4 \%$ of direct National Health Service (NHS) costs (notwithstanding costs to social services, patients and carers).

Factors responsible for suboptimal BP control include those due to patients, physicians and the health system. ${ }^{6}$ The key patient factors are adherence to medication and other health behaviours. ${ }^{7} 8$ Clinical inertia is another key issue, whereby clinicians fail to intensify treatment, despite evidence of inadequate control. It is estimated that clinical inertia may account for almost $20 \%$ of the variance in BP control, ${ }^{9}$ with evidence suggesting that treatment intensification fails to occur in nearly half $(45 \%)$ of consultations in which patients had a single BP reading above target, and around a third $(36 \%)$ with two successive readings above target. ${ }^{10}$ Lack of intensification may be due, in part, to the "white-coat effect', where BP readings in clinic consultations are elevated in comparison to self-monitored home BP readings; ${ }^{11} 12$ awareness that clinic readings may be inaccurate may contribute to clinician and patient reluctance to implement medication changes. Even with selfmonitoring of BP, clinical inertia can be profound. ${ }^{13}$

There is evidence that self-monitoring $\mathrm{BP}$ at home is useful in improving medication adherence, reducing therapeutic inertia and controlling $\mathrm{BP},{ }^{12}{ }^{14-17}$ and research by our team and others has shown that sustained reductions in BP can indeed be achieved by linking self-monitoring to preplanned medication titration when hypertension is uncontrolled. ${ }^{17-21}$ It can also detect and treat masked uncontrolled hypertension. ${ }^{22}$ While some evidence suggests that aggressive titration of antihypertensive medication without behavioural support is optimal for controlling $\mathrm{BP}^{23}$ further evidence suggests that greater reductions in BP may be achieved by the addition of behavioural support for selfmonitoring. ${ }^{192425}$ This may be further facilitated when combined with efficacious lifestyle changes, such as weight reduction, sodium restriction and increasing physical activity, ${ }^{26}{ }^{27}$ therefore HOME BP will combine all of these components.

\section{Rationale and risk-benefits for the current trial}

Increasingly widespread access to the internet and mobile phones $^{28} 29$ means that healthcare digital interventions (DIs) (also known as online interventions or computerbased health interventions within the literature) are accessible to the majority of patients and can be used to provide information and support at any time the patient needs it. ${ }^{30}$ DIs can empower patients by providing better access to personalised information and support for active involvement in treatment and self-management. ${ }^{30} \mathrm{~A}$ large meta-analysis found a small but significant positive effect of DIs on health-related behaviours, ${ }^{31}$ while a Cochrane review found evidence that computer-based health interventions for those with chronic health conditions significantly improved knowledge, health behaviours and clinical outcomes. ${ }^{32}$ DIs have the potential to make significant savings by automating routine aspects of patient education, monitoring and support, freeing up health professional resources for when patients most need them. ${ }^{33}$ There is accumulating evidence that DIs can deliver better and more efficient healthcare in the context of hypertension, ${ }^{34}{ }^{35}$ confirmed by a recent systematic review and meta-analysis undertaken by our team. ${ }^{36}$ Therefore, the HOME BP programme has been developed to incorporate previously identified effective intervention components (self-monitoring, preplanned medication titrations and behavioural support) delivered in an acceptable and feasible online format.

\section{Study aims and research questions}

The primary aim of the HOME BP trial is to assess the feasibility, acceptability, effectiveness and cost-effectiveness of adding the HOME BP intervention (comprising the HOME BP online DI, self-monitoring, medication titration and lifestyle interventions with nurse support) into primary care for the self-management of hypertension in comparison to usual care.

Main research question:

- Does the HOME BP intervention to assist self-monitoring and self-management of uncontrolled hypertension result in greater control of SBP after 1 year?

Secondary research questions:

- Does the HOME BP intervention to assist selfmonitoring and self-management of uncontrolled hypertension result in greater control of SBP and diastolic blood pressure (DBP) after 6 months and DBP after 1 year?

- Is the HOME BP intervention more cost-effective than usual care in managing poorly controlled hypertension in primary care?

Process analysis research questions:

- Which factors are related to patient engagement and adherence to HOME BP?

- Which factors are related to patient medication adherence and uptake of recommended medication titrations?

- Does the inclusion of lifestyle change choices and behavioural support result in engagement with lifestyle change?

- Is the HOME BP intervention acceptable to patients when integrated into routine practice?

- What are patient and healthcare practitioner views and experiences of the HOME BP intervention and its addition into primary care for the self-management of poorly controlled hypertension?

\section{METHODS AND ANALYSIS}

Study design and setting

The HOME BP intervention for the self-management of high $\mathrm{BP}$ consists of the integrated patient and healthcare 
practitioner HOME BP online DI, BP self-monitoring, health professional directed and supervised titration of antihypertensive medication and user-selected lifestyle modifications, for people with uncontrolled essential hypertension. The HOME BP study is a pragmatic, randomised controlled trial comparing the HOME BP intervention with optional nurse support to a control group receiving usual hypertension care within a UK primary care setting.

\section{Study participants}

Identification, follow-up and non-participation

Eligible participants will be identified from electronic database searches of clinical systems of collaborating general practices. Records of potentially eligible patients will be further screened by general practitioners (GPs) to remove participants known to meet exclusion criteria. Remaining potentially eligible patients will be invited by letter from the practice to attend a baseline clinic to establish eligibility, take consent, collect baseline data and randomise consented patients. Individuals not wanting to take part will be provided with a form to return should they wish to decline to take part in the trial. This will ask for basic demographic information and their reasons for declining, including an option not to give a reason if they prefer not to. The practice nurse (or other practice member as delegated by the lead GP) will have the option to follow-up invited patients by telephone after 1 month without response. All patient identification prior to consent will be conducted independently of the research team to maintain confidentiality.

\section{Eligibility criteria}

Eligibility criteria will aim to capture adult patients with treated but uncontrolled hypertension managed in primary care who will be aged 18 or over, have a Read code for hypertension in the clinical record (confirming hypertension), a current prescription of antihypertensive medication and a mean BP reading (calculated from the second and third BP readings) of $>140 / 90 \mathrm{~mm} \mathrm{Hg}$ taken during a baseline clinic appointment. Eligible patients will also be required to have access to the internet and be able to comprehend the website.

\section{Exclusion criteria}

Exclusion criteria will include an inability to self-monitor (eg, diagnosis of dementia), a mean baseline BP reading of $>180 / 110 \mathrm{~mm} \mathrm{Hg}$ (stage 3 hypertension requiring more urgent intervention than would be available in the trial), hypertension not managed by family doctor (the trial facilitates primary care titration of medication), prescribed more than three antihypertensive medications (ie, resistant hypertension), chronic kidney disease (CKD) stage 4-5 or CKD stage 3 not managed by the GP (likely to be under specialist care and requiring different BP targets), terminal disease or other condition which in the opinion of the family doctor makes them inappropriate to take part, pregnant or breast feeding, a history of proteinuria (albumin creatinine Ratio (ACR) $>30 \mathrm{mg} / \mathrm{mmol}$ ) (different BP targets), postural hypotension ( $>20 \mathrm{~mm} \mathrm{Hg}$ systolic drop after $1 \mathrm{~min}$ standing) (for whom intensification of BP medication may be inappropriate), atrial fibrillation (self-monitoring with oscillometric equipment not suitable), an acute cardiovascular event in the previous 3 months (BP and antihypertensive medication may not be stable) or a household member already enrolled on the study (to avoid bias). Participants who are involved in any other BP research will not be able to take part in the study.

\section{Randomisation}

Participants will be randomised in a 1:1 ratio to receive either usual care or the HOME BP intervention with optional nurse support using the HOME BP online system. Minimisation will be used taking into account participants' baseline SBP, age, diabetes status and practice. Patients are randomised to the optimal group $80 \%$ of the time. Any random numbers the minimisation routine might need will be computer generated, therefore bypassing study team involvement.

\section{Participant flow through the study}

At baseline, all patients will receive a consultation where informed consent will be taken, as well as clinical measures (confirmation of raised BP, height, weight) and medical history. Hypoglycaemic episodes in the last 6 months will be recorded for diabetic patients. BP will be measured after 5 min rest with a validated electronic automated sphygmomanometer (BP TRU BPM 200). Participants will be seated, and BP will be measured in their left arm unless there is a reason not to (principally lymphoedema or other medical condition). Six BP readings will be taken at intervals of $1 \mathrm{~min}$, with the mean of the second and third readings used for the primary outcome. Patients will complete demographic measures and baseline assessments at home via HOME BP online, following which automatic allocation to intervention or usual care group will be triggered. Patients will be immediately notified of their allocation through HOME BP online, confirmed by email or letter. Practice staff will also be notified of patient group allocation by email. Participants will be informed that the research team will have access to their personal and study data to stay in touch with them throughout the study.

Following randomisation, all patients will receive a $\mathrm{BP}$ medication review with a trained HOME BP prescriber (one or more GPs or nurse prescriber's nominated by the practice). For patients allocated to the intervention group, prescribers will be asked to select and agree an individualised medication titration plan (including three potential medication changes should BP remain above target). Participants allocated to usual care will receive a routine hypertension medication review. None of the study participants (patients or healthcare practitioners) will be blinded to group allocation. At 6-months and 
12 months after randomisation, all patients will be invited to a follow-up appointment with an independent research nurse. This may take place within the clinic or at a participant's home. Patients will be asked to not reveal their group allocation at this appointment, as research nurses completing the 6-month and 12-month follow-up appointments will be blinded to the patient's group. All follow-up BP measurements will be recorded no earlier than 2 weeks before the follow-up date, and no later than 4 weeks following the follow-up date at the 6-month follow-up. The follow-up period will be extended up to a maximum of 8 weeks for the 12-month follow-up. Patient BP readings and clinical measures will be taken in the same way as baseline and follow-up questionnaires completed through HOME BP online. Participant flow through the trial is outlined in figure 1. Participants will be withdrawn from the study if they become no longer eligible to participate (eg, due to pregnancy). Participants who choose not to continue with the study will be offered the opportunity to continue self-monitoring only rather than withdrawing completely from the study, and asked if they would be prepared to attend follow-up appointments.

\section{Sample size consideration}

A sample size of 244 patients per group is required in order to have $90 \%$ power to detect a difference in SBP of $5 \mathrm{~mm} \mathrm{Hg}$ (SD $17 \mathrm{~mm} \mathrm{Hg}$ ) between intervention and control groups, based on the findings from the TASMINH-2 study. ${ }^{20}$ Allowing for a $15 \%$ participant drop out, we will seek to recruit 287 participants per condition, resulting in a total sample size of 574 participants.

\section{Recruitment}

On the basis of our pilot work, and previous experience from hypertension self-management interventions, ${ }^{21}$ we estimate an approximate recruitment rate of $4 \%$ from the practice invite mail-out. Assuming an average practice list size of 5000, with a prevalence of hypertension of $13 \%$, it is estimated that $\sim 75 \%$ of the hypertension register will be excluded based on last BP reading and other practice exclusions. We estimate that $\sim 15 \%$ of patients invited will attend a baseline screening appointment, with $\sim 50 \%$ ineligible due to low BP readings during the screening appointment. We also estimate that a further $50 \%$ may decide not to participate due to the online format of the intervention. Around 95 practices are therefore likely to be required in order to recruit the target number of participants $(\mathrm{n}=574)$.

\section{HOME BP intervention}

The HOME BP intervention consists of HOME BP online (for patients and healthcare professionals), BP self-monitoring, medication titration procedures and optional behavioural support. Each of these components is described below, guided by the TIDieR checklist where possible. ${ }^{37}$ The intervention was developed using a theory-based, evidence-based and person-based approach, ${ }^{38}$ which is fully described elsewhere (article in submission).

\section{HOME BP online: patient behavioural components}

HOME BP online will use behavioural techniques to build patient motivation for BP self-monitoring, medication adherence and medication titration (Band R, Bradbury K, Morton K, et al. Intervention planning for a digital intervention for self-management of hypertension: a theory-, evidence- and person-based approach. Implement Sci. In submission). This will include presenting the health-related benefits of self-monitoring and reducing BP through medication titration procedures. Positive patient outcome expectancies will be encouraged by outlining ways that using the HOME BP intervention may reduce the chances of experiencing negative health consequences associated with hypertension. Common patient concerns regarding medication side effects are also addressed.

Patients will be instructed how to correctly undertake self-monitoring to promote patient self-efficacy. An Omron M3 monitor will be given to intervention group participants, and training provided through HOME BP online using a demonstration video. Participants will be asked to rehearse self-monitoring for a minimum of 7 days and enter their practice readings into HOME BP online before they are able to undertake study procedures. Participants will be able to print off tables to record each day's readings off-line and then enter all seven readings at one time (see figure 2 for screenshots of the intervention pages). Patients will also be provided with examples of successful hypertension management using HOME BP procedures to further increase selfefficacy for BP management.

Nine weeks after participants have been allocated to HOME BP, a behaviour change choice tool outlining the specific benefits of user-selected lifestyle modifications targeting healthy eating, physical activity, losing weight and salt and alcohol reduction will be available within HOME BP online. Users will be have the option of reading guidance about the benefits and commitments of each lifestyle change, so that they can choose the one (or more) which would be best for them. Patients will be reassured that additional behavioural support will be provided by their practice HOME BP support provider (usually a nurse or healthcare assistant) for selfmonitoring or lifestyle modifications, if required. The rationale for providing these options 9 weeks after the intervention began was to avoid making multiple behaviour changes ${ }^{39}$ until participants had an opportunity to form habits around the key target behaviours of self-monitoring and medication adherence. ${ }^{40}$

\section{HOME BP online: healthcare practitioner behavioural components}

HOME BP online will build healthcare practitioner motivation, knowledge, skills, positive outcome expectations and self-efficacy for study procedures, particularly 


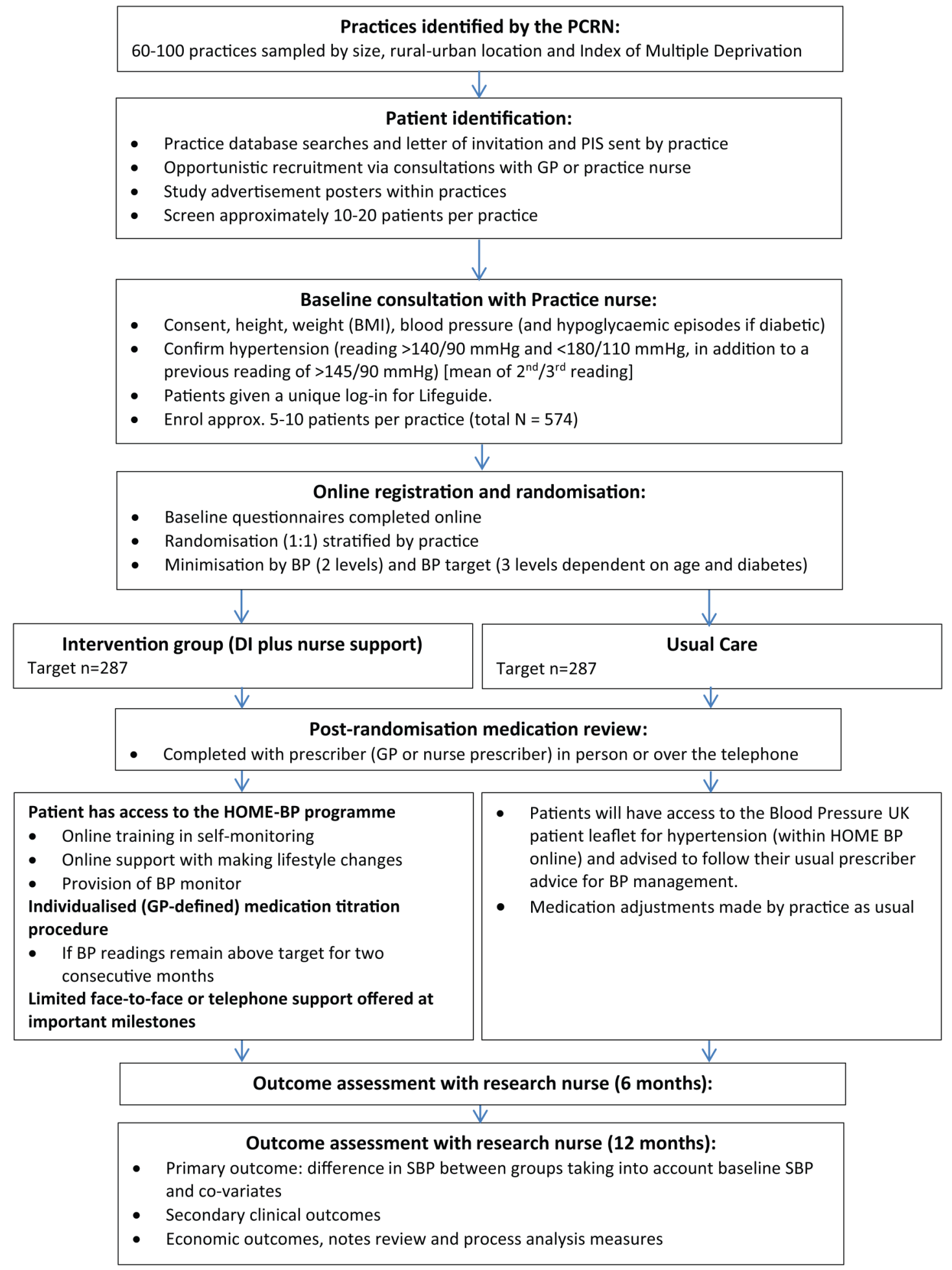

Figure 1 Participant flow through the HOME BP study. GPs, general practitioners; HOME BP, Home and Online Management and Evaluation of Blood Pressure.

medication titration. Healthcare practitioner self-efficacy will be promoted by addressing concerns regarding patient titration acceptance; the reliability of home blood-pressure readings and study procedures as an effective tool for overcoming clinical inertia for patients and practitioners will be outlined. Evidence demonstrating the health-related benefits of the medication titration procedure will be presented, in addition to evidence indicating that the medication escalation procedures do not result in increased patient side effects. ${ }^{14} 20{ }^{21}$ Users will be provided with information about the study procedures specific to their role (ie, prescriber or support provider) and information relating to study safety procedures. 
Figure 2 Screenshots of HOME BP pages. HOME BP, Home and Online Management and Evaluation of Blood Pressure.
Your Personal Blood Pressure

Management Programme

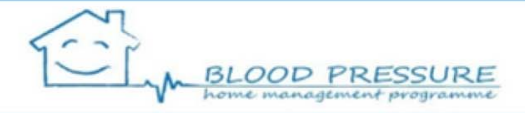

Taking your own blood pressure

Now that you have watched the video, you can take out your BP monitor and try

following these simple steps yourself.

1) You will need to fit the cuff before you turn the monitor on.

First check that the end of the grey rubber tube from the cuff is plugged into the small hole on the left hand side of the machine.

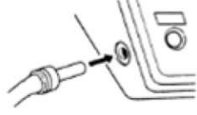

2) Sit comfortably with both feet flat on the floor

do not cross your legs). Slip the cuff onto the arm

that you don't use for writing, so that the air tube is

on the inside of your wrist and at the bottom

of the cuft, and isn't squashed.

3) Wrap the cuff around the bare skin of your upper arm so that the bottom of the cuff is half an inch (or $1-2 \mathrm{~cm}$ ) above your elbow.

Make sure there isn't a gap between the cuff and your arm and fasten it using the Velcro.

The most common reason for inaccurate readings is that people have their cuff too loose so that it doesnt measure their BP properly.

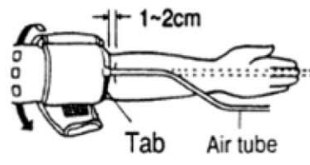

Your Personal Blood Pressure Management Programme

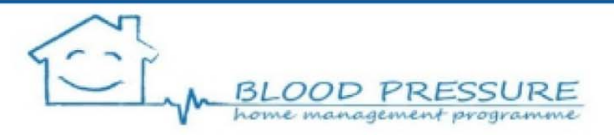

\section{BP management table for practice readings}

Five Easy Steps:

1. Please enter your BP readings taken during your week of practicing at home in order with the earliest reading first.

2. You can use the drop-down menus to choose the right option, or you can type the readings in if you prefer

3. The systolic reading should be the higher of your two readings

4. Remember, you only need to enter the second of your two BP measurements from each day.

5. Please double check your readings are correct as once you click submit, they cannot be changed.

\begin{tabular}{|c|c|c|c|}
\hline & $\begin{array}{l}\text { Time of day } \\
\text { (am/pm) }\end{array}$ & $\begin{array}{l}\text { Systolic reading } \\
\text { (SYS) }\end{array}$ & $\begin{array}{l}\text { Diastolic reading } \\
\text { (D|A) }\end{array}$ \\
\hline Day 1 & Please select $\mathrm{r}$ & $-\quad \cdot$ & $-\quad \cdot$ \\
\hline Day 2 & Please select ${ }^{-}$ & $-\cdot$ & - \\
\hline Day 3 & Please select $*$ & $-\cdot$ & $-\cdot$ \\
\hline Day 4 & Please select - & $-\quad r$ & $-\cdot$ \\
\hline Day 5 & Please select ${ }^{*}$ & $-\cdot$ & - \\
\hline
\end{tabular}


Patients and healthcare practitioners will be able to re-access their specific behavioural intervention components from HOME BP online at any time throughout the study.

\section{Self-monitoring procedures}

Patients will be advised to take two morning BP readings for 7 days each month and in each case to use the second reading (as per the TASMINH2 and TASMIN-SR studies). ${ }^{20}{ }^{21}$ Automated email prompts will be sent to participants as reminders to begin BP self-monitoring. Patients will need to have seven readings to enter into HOME BP online, but will be able to flexibly complete their readings over a 2-week period if required. Following the completion of each set of seven readings, patients will have access to the behavioural intervention components described above, but will not enter further $\mathrm{BP}$ readings for further 4 weeks.

\section{BP targets}

BP targets will be set in line with the National Institute for Health and Care Excellence (NICE) hypertension guidelines, ${ }^{41}$ with adjustment by $5 / 5 \mathrm{~mm} \mathrm{Hg}$ for home readings. ${ }^{42}$ People under 80 without diabetes will have BP targets of $<135 / 85 \mathrm{~mm} \mathrm{Hg}$, while patients aged 80 and over without diabetes will have a target BP of $<145$ / $85 \mathrm{~mm} \mathrm{Hg}$. Target BP for all patients with diabetes will be $<135 / 75 \mathrm{~mm} \mathrm{Hg}$ (those with proteinuria and therefore lower targets are excluded). After the seven readings are entered in to HOME BP online, the mean BP will be calculated and feedback provided to the patient and the healthcare practitioners according to a traffic light system (developed from that used in previous medication titration procedures)..$^{20}{ }^{21}$ These readings will be used to inform the medication titration procedure.

\section{Medication titration procedures}

All HOME BP prescribers will receive a copy of the NICE hypertension guidelines ${ }^{41}$ and will complete an individualised patient medication review at baseline for intervention group participants, where three potential medication changes will be selected and agreed with the patient. If a patient's mean BP readings are above target for two consecutive months, the medication titration procedure will be initiated by HOME BP online. Patients and practice staff will be informed by email that a medication change is recommended. The prescriber will be asked to implement the preplanned medication change and confirm this with the patient (or explain the reasons for nonimplementation, if applicable). If patients decide not to initiate a medication change, the prescriber will receive information provided by the patient outlining the reasons for non-titration. When a recommended medication change is not initiated, an above target reading at the subsequent $\mathrm{BP}$ entry will again prompt a medication change recommendation from HOME BP online.

If patients take two $\mathrm{BP}$ readings that are too high $(>180 / 110 \mathrm{~mm} \mathrm{Hg})$ or low $(\mathrm{SBP}<100 \mathrm{~mm} \mathrm{Hg}$ ), they will be asked to repeat the measurement after $5 \mathrm{~min}$. If readings are still too high or too low, the HOME BP healthcare practitioners will be alerted by email and patients will be advised to make an appointment with the prescriber in the usual way within 3 days. Decisions about patients' medication will remain at the prescriber's discretion at all times. If a mean target $\mathrm{BP}$ (ie, below target but SBP above $100 \mathrm{~mm} \mathrm{Hg}$ ) is entered into HOME BP online for three consecutive months, patients will be advised to reduce $\mathrm{BP}$ monitoring to once every 8 weeks as their BP is currently well controlled. This will revert to monthly should subsequent mean $\mathrm{BP}$ rise above target.

\section{HOME BP behavioural support}

Optional additional behavioural support for selfmonitoring and lifestyle modifications will be available to all patients via the practice support providers, who will be trained in applying the CARE (Congratulate, Ask, Reassure, Encourage) approach (paper outlining this approach in HOME BP forthcoming). A face-to-face appointment is offered to all participants at 4 weeks following randomisation and 1 week after any lifestyle modifications are introduced, with an option for remote telephone or email support if preferred. A maximum of six face-to-face support sessions will be available if patients experience difficulties associated with selfmonitoring or lifestyle change, and patients will have the option to email their support provider through HOME BP online at any point. Template-guided motivating and reassuring support messages will be sent by email from the support provider to all patients every 4 weeks to encourage and reinforce patient adherence to monitoring, medication regimes and lifestyle changes (where applicable).

\section{Monitoring adherence}

Automated email reminders will be sent to patients and healthcare practitioners at key milestones to promote adherence to study procedures. Adherence to titration procedures will also be monitored by the healthcare practitioners and study team to ensure changes have been enacted (or to understand why not). At the end of the study, each HOME BP supporter will return a copy of their support log. An audit of a selection of BP monitors will be conducted.

\section{Usual care}

Patients allocated to usual care will have access to the information provided in the Blood Pressure UK patient leaflet for hypertension through HOME BP online. They will receive routine hypertension care, with appointments and medication changes made at the GP's discretion.

\section{Outcomes}

The primary outcome of the trial will be difference in office SBP ( $\mathrm{mm} \mathrm{Hg}$, mean of second/third readings) at 
12-month follow-up between the intervention and control groups adjusting for baseline BP, practice, BP target levels and gender. These will be entered and stored on an independent, secure server.

Secondary outcomes (also adjusted for baseline and covariates where appropriate) will comprise:

- Difference in office SBP between intervention and control groups at 6-month follow-up.

- Difference in office DBP between intervention and control groups at 6-month and 12-month follow-up.

- Difference in office SBP and DBP at 6 and 12 months using the mean of the second to sixth BP readings.

- Difference in health-related quality of life measured using EQ-5D-5L. ${ }^{43}$

- Difference in patient enablement measured using the patient enablement instrument. ${ }^{44}$

- Economic modelling (including cost of equipment and drugs, patient and healthcare professional time and support provision).

Process evaluation measures will include:

- Medication beliefs measured using the Beliefs about Medicines Questionnaire (BMQ) ${ }^{45}$ and medication adherence using the Medication Adherence Report Scale (MARS). ${ }^{46}$

- Patient perceived side effects measured using the symptom subscale of the Illness Perception questionnaire (IPQ-R) hypertension. ${ }^{47}$

- Patient self-efficacy. ${ }^{48}$

- Medication use (number and defined daily doses). ${ }^{49}$

- Patient weight measured within clinic and selfreported lifestyle behaviours.

- Prescriber and support provider self-efficacy and outcome expectations.

- Prescriber and support provider confidence in the acceptability of the intervention.

Intervention group only:

- DI website usage and engagement, including lifestyle choice, usage and progress.

- Patient BP monitoring adherence measured by the HOME BP online system.

- Adherence to recommended medication changes.

- Usage of optional support provision.

- Prescriber initiation of titration procedures.

\section{Adverse events}

HOME BP healthcare practitioners will inform investigators of all patient adverse events occurring throughout the trial. The assessment of whether or not an serious adverse event (SAE) is an expected consequence of receiving the intervention will be provided by the chief investigator (or clinical reviewer delegate), it will not be provided by the investigator responsible for the care of the participant.

\section{Study end points}

Participants in the intervention and control groups will be invited to appointments with an independent research nurse for assessment of primary and secondary end points at 6 months and 12 months following entry into the study. These appointments will be conducted in the same way for both groups. Any other contacts with the GP practice (outside of the study) will be recorded in the medical notes and reported for the economic analysis at the end of the trial (based on blind review of patient medical notes). Only members of the study research team involved in the data collection, and data analysis will have access to the final trial data set.

\section{Measures}

See table 1 for full details of study measures.

\section{Statistical analysis}

The principal analysis will use raw and adjusted data and will be performed at the end of the trial after all data have been collected. The primary analysis will use general linear modelling to compare intervention and usual care SBP at follow-up adjusting for baseline BP, practice (as a random effect), BP target levels and gender. Analyses will be on an intention to treat basis with imputation. Secondary analyses will be reported for complete cases. No interim analyses will be performed. A sensitivity analysis will examine the potential effect of missing data using multiple imputation. Planned subgroup analyses will be of BP target groups, older versus younger (65 as threshold), men versus women and better controlled at baseline versus worse controlled at baseline (median SBP). Secondary analyses will use similar techniques to assess differences between groups in SBP at 6 months, DBP at 6 months and 12 months, medication use and quality of life. Additional analyses will examine patient and healthcare professional adherence to advice and study processes, website usage and support provision. Process analyses will be reported elsewhere, but will assess the impact of patient enablement and self-efficacy, symptom perceptions and medication beliefs on outcomes. These analyses will also examine the relationship between support uptake, engagement with healthy behaviour change and outcomes.

\section{Economic analysis}

TASMINH2 ${ }^{20}$ resulted in a $5.4 \mathrm{~mm} \mathrm{Hg}$ reduction in BP in self-management compared with usual care which was cost-effective with $£ 1.6 \mathrm{k}$ per QALY for men and $£ 4.9 \mathrm{k}$ for women. Thus if HOME BP results in the hypothesised difference of $5 \mathrm{~mm} \mathrm{Hg}$, then using the same cost-effectiveness model, it will be highly cost-effectiveunless of course the costs are much greater. The intervention cost in TASMINH2 was $£ 105$, which included the BP monitor, tele-monitoring connection and training costs (see table 2 in Kaambwa et $a \rho^{50}$ ). Key differences between the interventions in HOME BP and TASMINH2 have to do with the use of behavioural techniques in the former, including guidance about lifestyle change and support in making such changes as well as recommendations to change medications with recording of whether such changes were made and further 
Table 1 Measures and schedule of observations within the HOME BP trial

\begin{tabular}{|c|c|c|c|c|c|}
\hline \multirow[b]{2}{*}{ Measure } & \multicolumn{5}{|l|}{ Time point } \\
\hline & Baseline/screening & Visit 1 & Visit 2 & Intervention group only & Usual care group only \\
\hline Month & 0 & 6 & 12 & $(0-12)$ & 12 \\
\hline Patient sociodemographic measures & $\mathrm{x}$ & & & & \\
\hline Frequency of previous self-monitoring & $\mathrm{x}$ & & & & $\mathrm{x}$ \\
\hline \multicolumn{6}{|l|}{ Clinical measures } \\
\hline Systolic BP (SBP) & $\mathrm{x}$ & $\mathrm{x}$ & $\mathrm{x}$ & & \\
\hline Diastolic BP (DBP) & $\mathrm{x}$ & $\mathrm{x}$ & $\mathrm{x}$ & & \\
\hline Weight $(\mathrm{kg})$ & $\mathrm{x}$ & & $\mathrm{x}$ & & \\
\hline Height $(\mathrm{cm})$ & $\mathrm{x}$ & & & & \\
\hline Diabetes status & $\mathrm{x}$ & & & & \\
\hline Hypoglycaemic episodes in last 6 months & $\mathrm{x}$ & $\mathrm{x}$ & $\mathrm{X}$ & & \\
\hline Medication changes (prescriptions issued) & & & $X(N R)$ & & \\
\hline Consultations & & & $X(N R)$ & & \\
\hline \multicolumn{6}{|l|}{ Patient self-report measures } \\
\hline Patient enablement instrument & $\mathrm{x}$ & & $\mathrm{x}$ & & \\
\hline Patient self-efficacy & $\mathrm{x}$ & $\mathrm{x}$ & $\mathrm{x}$ & & \\
\hline Beliefs about medication (BMQ) & $\mathrm{x}$ & $\mathrm{x}$ & $\mathrm{x}$ & & \\
\hline Medication adherence (MARS) & $\mathrm{x}$ & & $\mathrm{x}$ & & \\
\hline Patient perceived side effects (IPQ-R hypertension: symptoms subscale) & $\mathrm{x}$ & & $\mathrm{x}$ & & \\
\hline Changes to lifestyle behaviours & & & $\mathrm{x}$ & & \\
\hline \multicolumn{6}{|l|}{ Patient objectively recorded measures } \\
\hline Website usage & & & & $X^{*}$ & \\
\hline Usage of hypertension pages & & & & $X^{*}$ & \\
\hline \multicolumn{6}{|l|}{ Monitoring of blood pressure } \\
\hline Self-reported SBP & & & & $X^{*}$ & \\
\hline Self-reported DBP & & & & $X^{*}$ & \\
\hline Medication titration recommended & & & & $X^{*}$ & \\
\hline Titration uptake & & & & $X^{*}$ & \\
\hline Reasons for non-titration & & & & $X^{*}$ & \\
\hline Usage of lifestyle pages & & & & $X^{*}$ & \\
\hline Choice of lifestyle changes & & & & $X^{*}$ & \\
\hline Reported progress on lifestyle change (eg, weight change) & & & & $X^{*}$ & \\
\hline \multicolumn{6}{|l|}{ HCP objectively recorded measures } \\
\hline Usage of training pages (prescriber guide) & & & & $\mathrm{x}$ & \\
\hline Emails sent to the patient & & & & $\mathrm{x}$ & \\
\hline Titration procedure compliance & & & & $\mathrm{x}$ & \\
\hline Support provision & & & & $\mathrm{x}$ & \\
\hline \multicolumn{6}{|l|}{ HCP self-report measures } \\
\hline Self-efficacy and outcome expectations & $x \dagger$ & & & & \\
\hline Confidence in the acceptability of the intervention (for patients) & $x \dagger$ & & & & \\
\hline
\end{tabular}




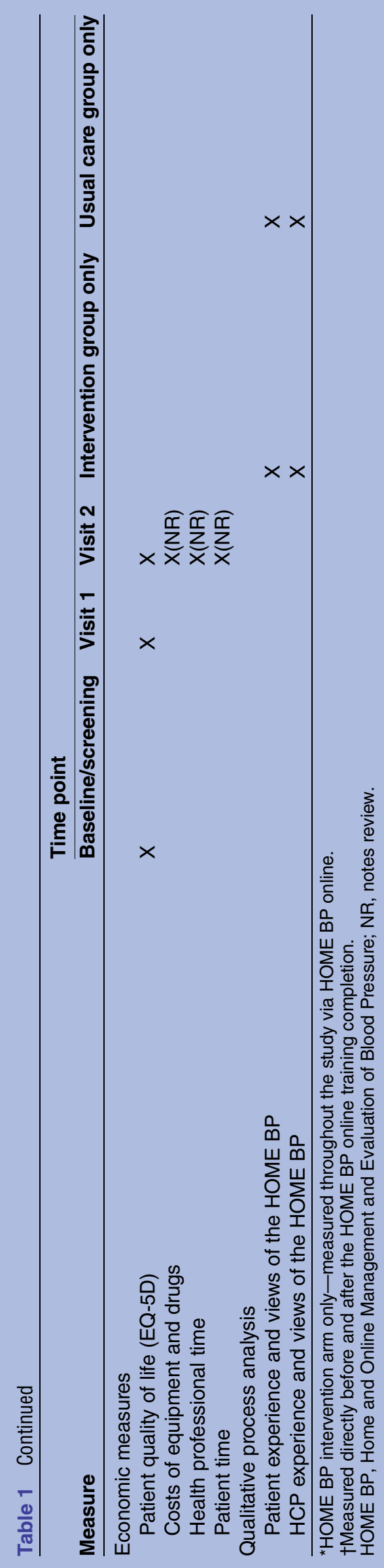

reminders if not. HOME BP thus tests the intervention as delivered digitally and the additional value of behavioural support in self-management. To facilitate costing of the behavioural add-ons to self-management, the costing of the intervention in HOME BP as far as possible will separate these elements.

\section{Economic analysis plan}

A within trial analysis will estimate cost per unit reduction in SBP, the primary outcome, with adjustments in that outcome as described in the 'Statistical analysis' section, including subgroups. Costs will include those due to the intervention, as well as those due to changes in medication, use of other relevant NHS resources and any costs borne by patients such as in diet and lifestyle. NHS and societal costs will be estimated and uncertainty explored in sensitivity analyses. To facilitate analysis of the difference between the intervention in HOME BP and that of self-management, the components and the cost of the intervention in HOME BP will be compared with that of TASMINH2.

These short-term results will provide input to a longterm cost-effectiveness model, based on that developed for TASMINH2, updated by subsequent work as relevant. Since these studies all show reductions in BP as cost reducing or highly cost-effective, this modelling will address the cost-effectiveness of the HOME BP-intervention versus usual care but also of the addition of the behavioural/digital element relative to paper-based self-management without that element. The HOME BP trial will collect data on numbers and types of antihypertensive prescriptions between arms.

\section{Qualitative substudy}

The qualitative interviews will seek to provide an in-depth understanding of the factors that may facilitate or diminish the acceptability of the HOME BP intervention, and adherence to implementation from the perspective of patients and healthcare professionals using HOME BP. Interviews and focus groups will be undertaken alongside participation and after participants finish the HOME BP intervention for patients in the intervention and usual care groups. Purposive sampling will be used to select patients from the intervention group by age, gender, socioeconomic status and level of engagement with the website to allow for a wide range of views and experiences of the HOME BP online and the HOME BP intervention. Open-ended inductive questions will be used to elicit user perspectives and experiences of the intervention and the support provided, allowing participants to freely describe their experiences and views in their own way and to focus on whatever is most salient to them. Focus group discussions and interviews will also be conducted with healthcare professionals who have been involved in the trial procedures or intervention delivery. Interviews and focus groups will be audio-recorded, fully transcribed and analysed thematically. 


\section{ETHICS AND DISSEMINATION}

\section{Ethical approval}

Ethical approval for the HOME BP study has been obtained from the South Central-Hampshire A Ethics Committee (reference: 15/SC/0082). R\&D approvals have been obtained from all relevant Clinical Research Networks. All substantial amendments must be approved by the University Ethics Committee and NHS Ethics Committee responsible for the trial, in addition to approval by NHS R\&D. Investigators are kept up to date with relevant changes via regular management group meetings.

\section{Data monitoring}

The Programme Steering Committee is responsible for ensuring programme adherence to the protocol, and adherence to the requirements of the Guidelines for Good Clinical Practice. It was decided by the Steering Committee that a data monitoring committee was not required. The trial may be subject to inspection and audit by University of Southampton, under their remit as sponsor, the trial coordinating centre as the Sponsor's delegate and other regulatory bodies.

\section{Dissemination}

Dissemination of our research will be via multiple pathways. We will submit the primary, secondary and tertiary study results for publication in highly cited and open access peer-reviewed journals and present findings at national and international conferences. We will also provide summaries of HOME BP findings to professional societies (such as British Hypertension Society), patient groups (Blood Pressure UK), participants, NHS organisations and healthcare providers. We will also disseminate to the public through regular press releases and to stakeholders through interactive workshops.

If cost-effective, the HOME BP intervention will be disseminated for clinical use by University of Southampton or a suitable, appropriate licensed partner. Following the Behavioral Intervention Technology model, ${ }^{51}$ we anticipate that future technology enhancements in mode of delivery of the intervention will be possible and desirable without the need to re-evaluate the essential behaviour support content of the intervention. For example, as smartphone use becomes more prevalent in the target population it will be easy to transfer the content of HOME BP for delivery by smartphone, with automatic transfer of BP readings (which will reduce the potential for patient error).

\footnotetext{
Author affiliations

${ }^{1}$ Academic Unit of Psychology, University of Southampton, Southampton, UK ${ }^{2}$ Primary Care and Population Sciences, Faculty of Medicine, University of Southampton, Southampton, UK

${ }^{3}$ Faculty of Medicine, University of Southampton, Southampton, UK

${ }^{4}$ Nuffield Department of Primary Care Health Sciences, University of Oxford, Oxford, UK
}

Acknowledgements The initial development of the intervention, which lead to the application for, and subsequent funding of, the current Programme by NIHR PGfAR, was made possible by a pump priming grant from the NIHR School for Primary Care Care Research (Principal investigator: PL, co-applicants: RJM, LY).

Contributors $L Y$ developed the initial idea for the study and obtained funding in collaboration with RJM, PL, BS, JR, GLY and the DIPSS study team. All authors have contributed to the protocol development; RJM and PL provided clinical expertise and experience from the original TASMINH2 intervention; LY, $\mathrm{KB}, \mathrm{RB}$ and $\mathrm{KM}$ led the intervention development and trial preparations; BS led the statistical analysis and JR, GLY and SZ have led the health economics planning. RB wrote the initial draft supervised by $L Y$ and RJM, and all subsequent drafts were contributed to by all authors who have approved the final version.

Funding This protocol paper summarises independent research funded by the National Institute for Health Research (NIHR) under its Programme Grants for Applied Research (grant reference number RP-PG-1211-20001).

Disclaimer The views expressed are those of the author(s) and not necessarily those of the NHS, the NIHR or the Department of Health.

Competing interests RJM has received blood pressure monitoring equipment for research purposes from Omron and Lloyds Pharmacies and travel expenses and honoraria to speak at conferences from the American Society of Nephrology and Japanese Society of Hypertension.

Ethics approval South Central-Hampshire A ethics committee (reference: 15/SC/0082)

Provenance and peer review Not commissioned; externally peer reviewed.

Open Access This is an Open Access article distributed in accordance with the terms of the Creative Commons Attribution (CC BY 4.0) license, which permits others to distribute, remix, adapt and build upon this work, for commercial use, provided the original work is properly cited. See: http:// creativecommons.org/licenses/by/4.0/

\section{REFERENCES}

1. Kearney PM, Whelton M, Reynolds K, et al. Global burden of hypertension: analysis of worldwide data. Lancet 2005;365:217-23.

2. Craig R, Mindell J, eds. Health Survey for England 2011: Health, social care and lifestyles. Health and Social Care Information Centre Leeds, 2012.

3. Falaschetti E, Mindell J, Knott C, et al. Hypertension management in England: a serial cross-sectional study from 1994 to 2011. Lancet 2014;383:1912-19.

4. World Health Organization. Surveillance of chronic disease risk factors-country-level data and comparable estimates. The SuRF Report 2. InfoBase, 2005.

5. Law MR, Morris JK, Wald NJ. Use of blood pressure lowering drugs in the prevention of cardiovascular disease: meta-analysis of 147 randomised trials in the context of expectations from prospective epidemiological studies. BMJ 2009;338:b1665.

6. Ogedegbe G. Barriers to optimal hypertension control. J Clin Hypertens (Greenwich) 2008;10:644-66.

7. Grant RW, Pandiscio JC, Pajolek $\mathrm{H}$, et al. Implementation of a web-based tool for patient medication self-management: the Medication Self-titration Evaluation Programme (Med-STEP) for blood pressure control. Inform Prim Care 2012;20:57-67.

8. Halifax NV, Cafazzo JA, Irvine MJ, et al. Telemanagement of hypertension: a qualitative assessment of patient and physician preferences. Can J Cardiol 2007;23:591-4.

9. Okonofua EC, Simpson KN, Jesri A, et al. Therapeutic inertia is an impediment to achieving the healthy people 2010 blood pressure control goals. Hypertension 2006:47:345-51.

10. Guthrie B, Inkster M, Fahey T. Tackling therapeutic inertia: role of treatment data in quality indicators. BMJ 2007;335:542-4.

11. Clark CE, Horvath IA, Taylor RS, et al. Doctors record higher blood pressures than nurses: systematic review and meta-analysis. Br J Gen Pract 2014;64:e223-32.

12. Agarwal R, Bills JE, Hecht TJ, et al. Role of home blood pressure monitoring in overcoming therapeutic inertia and improving hypertension control: a systematic review and meta-analysis. Hypertension 2011;57:29-38. 
13. Asayama K, Ohkubo T, Metoki $\mathrm{H}$, et al., Hypertension Objective Treatment Based on Measurement by Electrical Devices of Blood Pressure (HOMED-BP). Cardiovascular outcomes in the first trial of antihypertensive therapy guided by self-measured home blood pressure. Hypertens Res 2012;35:1102-10.

14. Bray EP, Holder R, Mant $\mathrm{J}$, et al. Does self monitoring reduce blood pressure? Meta-analysis with meta-regression of randomized controlled trials. Ann Med 2010;42:371-86.

15. Watson AJ, Singh K, Myint-U K, et al. Evaluating a web-based self-management program for employees with hypertension and prehypertension: a randomized clinical trial. Am Heart $J$ 2012;164:625-31.

16. Ogedegbe G, Schoenthaler A. A systematic review of the effects of home blood pressure monitoring on medication adherence. J Clin Hypertens (Greenwich) 2006;8:174-80.

17. Fletcher BR, Hinton L, Hartmann-Boyce J, et al. Self-monitoring blood pressure in hypertension, patient and provider perspectives: a systematic review and thematic synthesis. Patient Educ Couns 2016;99:210-19.

18. Bosworth $\mathrm{HB}$, Olsen MK, McCant F, et al. Hypertension Intervention Nurse Telemedicine Study (HINTS): testing a multifactorial tailored behavioral/educational and a medication management intervention for blood pressure control. Am Heart J 2007:153:918-24.

19. Bosworth HB, Powers BJ, Olsen MK, et al. Home blood pressure management and improved blood pressure control: results from a randomized controlled trial. Arch Intern Med 2011:171:1173-80.

20. McManus RJ, Mant J, Bray EP, et al. Telemonitoring and self-management in the control of hypertension (TASMINH2): a randomised controlled trial. Lancet 2010;376:163-72.

21. McManus RJ, Mant J, Haque MS, et al. Effect of self-monitoring and medication self-titration on systolic blood pressure in hypertensive patients at high risk of cardiovascular disease: the TASMIN-SR randomized clinical trial. JAMA 2014;312:799-808.

22. Banegas JR, Ruilope LM, de la Sierra A, et al. High prevalence of blinded uncontrolled hypertension in people with treated hypertension. Eur Heart J 2014;35:3304-12.

23. Green BB, Cook AJ, Ralston JD, et al. Effectiveness of home blood pressure monitoring, web communication, and pharmacist care on hypertension control: a randomized controlled trial. JAMA 2008;299:2857-67.

24. Uhlig K, Patel K, Ip S, et al. Self-measured blood pressure monitoring in the management of hypertension: a systematic review and meta-analysis. Ann Intern Med 2013;159:185-94.

25. Margolis KL, Asche SE, Bergdall AR, et al. Effect of home blood pressure telemonitoring and pharmacist management on blood pressure control. A cluster randomized clinical trial. JAMA 2013;310:46-56

26. Dickinson HO, Mason JM, Nicolson DJ, et al. Lifestyle interventions to reduce raised blood pressure: a systematic review of randomized controlled trials. J Hypertens 2006;24:215-33.

27. Booth JN III, Levitan EB, Brown TM, et al. Effect of sustaining lifestyle modifications (nonsmoking, weight reduction, physical activity and mediterranean diet) after healing of myocardial infarction, percutaneous intervention or coronary bypass (from the REasons for Geographic and Racial Differences in Stroke Study). Am J Cardiol 2014;113:1933-40.

28. Ofcom. UK communications market report. UK mobile phone usage statistics, London: Ofcom, 2013.

29. Office of National Statistics. Statistical Bulletin: Internet Access for Households and Individuals. London, UK, 2013.

30. Bradbury K, Watts S, Arden-Close E, et al. Developing digita interventions: a methodological guide. Evid Based Complement Alternat Med 2014;2014:561320.

31. Webb TL, Joseph J, Yardley L, et al. Using the internet to promote health behavior change: a systematic review and meta-analysis of the impact of theoretical basis, use of behavior change techniques, and mode of delivery on efficacy. J Med Internet Res 2010;12:e4.
32. Murray E, Burns J, See TS, et al. Interactive health communication applications for people with chronic disease. Cochrane Database Syst Rev 2005(4):CD004274

33. McDermott MS, While A. Maximizing the healthcare environment: systematic review exploring the potential of computer technology to promote self-management of chronic illness in healthcare settings. Patient Educ Couns 2013;92:13-22.

34. Piette JD, Datwani H, Gaudioso S, et al. Hypertension management using mobile technology and home blood pressure monitoring: results of a randomized trial in two low/middle-income countries. Telemed J E Health 2012;18:613-20.

35. Nolan RP, Liu S, Shoemaker JK, et al. Therapeutic benefit of internet-based lifestyle counselling for hypertension. Can J Cardiol 2012;28:390-6.

36. McLean G, Band R, Saunderson K, et al., DIPSS co-investigators. Digital interventions to promote self-management in adults with hypertension systematic review and meta-analysis. J Hypertens 2016;34:600-12.

37. Hoffmann TC, Glasziou PP, Boutron I, et al. Better reporting of interventions: template for intervention description and replication (TIDieR) checklist and guide, 2014

38. Yardley L, Morrison L, Bradbury K, et al. The person-based approach to intervention development: application to digital health-related behavior change interventions. J Med Internet Res 2015; 17:e30.

39. Hyman DJ, Pavlik VN, Taylor WC, et al. Simultaneous vs sequential counseling for multiple behavior change. Arch Intern Med 2007;167:1152-8.

40. Gardner B, Lally P, Wardle J. Making health habitual: the psychology of 'habit-formation' and general practice. $\mathrm{Br} J$ Gen Pract 2012;62:664-6.

41. National Institute for Health and Care Excellence: Clinical Guidelines. Clinical Guideline 127 Hypertension: The clinical management of primary hypertension in adults. London: Royal College of Physicians (UK); 2011.

42. Williams B, Poulter NR, Brown MJ, et al., British Hypertension Society. Guidelines for management of hypertension: report of the fourth working party of the British Hypertension Society, 2004-BHS IV. J Hum Hypertens 2004;18:139-85.

43. Group TE. EuroQol-a new facility for the measurement of health-related quality of life. Health Policy 1990;16:199-208.

44. Howie JG, Heaney DJ, Maxwell M, et al. A comparison of a Patien Enablement Instrument (PEI) against two established satisfaction scales as an outcome measure of primary care consultations. Fam Pract 1998;15:165-71.

45. Horne R, Weinman J, Hankins M. The Beliefs about Medicines Questionnaire: the development and evaluation of a new method for assessing the cognitive representation of medication. Psychol Health 1999;14:1-24.

46. Horne R. Nonadherence to medication: causes and implications for care. In: Gard P, ed. A behavioural approach to pharmacy practice. Oxford: Blackwell, 2001:111-30.

47. Moss-Morris R, Weinman J, Petrie KJ, et al. The Revised Illness Perception Questionnaire (IPQ-R). Psychol Health 2002;17:1-16.

48. Bandura A. Health promotion from the perspective of social cognitive theory. Psychol Health 1998;13:623-49.

49. WHO Collaborating Centre for Drug Statistics Methodology. Guidelines for ATC classification and DDD assignment, 2015. Oslo, 2014.

50. Kaambwa B, Bryan S, Jowett S, et al. Telemonitoring and self-management in the control of hypertension (TASMINH2): a cost-effectiveness analysis. Eur J Prev Cardiol 2014;21: 1517-30.

51. Mohr DC, Schueller SM, Montague E, et al. The behavioral intervention technology model: an integrated conceptual and technological framework for eHealth and mHealth interventions. $J$ Med Internet Res 2014;16:e146. 